\title{
On rings of quotients of semiprime $\Gamma$-rings
}

\author{
Emine Koç and Öznur Gölbaşi
}




\title{
ON RINGS OF QUOTIENTS OF SEMIPRIME $\Gamma$-RINGS
}

\author{
EMINE KOÇ AND ÖZNUR GÖLBAŞI
}

Received 30 March, 2011

Abstract. In this paper, we investigate the rings of quotients of a semiprime $\Gamma$-ring.

2000 Mathematics Subject Classification: 16 Y99

Keywords: $\Gamma$-rings, prime $\Gamma$-rings, semiprime $\Gamma$-rings, ring of quotients

\section{INTRODUCTION}

Let $M$ be an abelian additive group whose elements are denoted by $a, b, c, \cdots$ and let $\Gamma$ another abelian additive group whose elements are $\gamma, \beta, \alpha, \cdots$. Suppose that $a \gamma b$ is defined to be an element of $M$ and that $\gamma a \beta$ is defined to be an element of $\Gamma$ for every $a, b, \gamma$ and $\beta$. If the products satisfy the following three conditions:

(i) $(a+b) \gamma c=a \gamma c+b \gamma c, a(\alpha+\beta) b=a \alpha b+a \beta b, a \gamma(b+c)=a \gamma b+a \gamma c$,

(ii) $(a \gamma b) \beta c=a(\gamma b \beta) c=a \gamma(b \beta c)$,

(iii) $a \alpha b=0$ for all $a, b \in M$ implies $\alpha=0$,

then $M$ is called a $\Gamma$-ring in the sense of Nabusawa [7].

Barnes approached $\Gamma$-ring in a different way compared to that of Nobusawa and he defined the concept of $\Gamma$-rings.

Let $M$ and $\Gamma$ be additive abelian groups. If there exits a mapping of $M \times \Gamma \times M$ to $M$ (the image of $(a, \gamma, b) a, b \in M, \gamma \in \Gamma$ is denoted by $(a \gamma b)$ ) satisfying for all $a, b, c \in M, \alpha, \beta \in \Gamma$ :

(i) $(a+b) \gamma c=a \gamma c+b \gamma c, a(\alpha+\beta) b=a \alpha b+a \beta b, a \gamma(b+c)=a \gamma b+a \gamma c$,

(ii) $(a \gamma b) \beta c=a \gamma(b \beta c)$,

then $M$ is called a $\Gamma$-ring in the sense of Barnes [2].

Throughout the present paper, the symbol $(\Gamma, M)_{N}$ that stands for $M$ is the $\Gamma$-ring in the sense of Nabusawa, and the symbol $(\Gamma, M)_{B}$ that stands for $M$ is the $\Gamma$-ring in the sense of Barnes. In [5], we have shown that for all $(\Gamma, M)_{B}$ there exists $\Gamma^{\prime}$ as an additive group such that $\left(\Gamma^{\prime}, M\right)_{N}$. Therefore, if $\Gamma$-ring in the sense of Barnes, then $\Gamma^{\prime}$-ring in the sense of Nabusawa.

Let $M$ be a $\Gamma$-ring in the sense of Nabusawa. A right (resp. left) ideal of $M$ is an additive subgroup of $U$ such that $U \Gamma M \subseteq U$ (resp. $M \Gamma U \subseteq U$ ). If $U$ is both a right and left ideal of $M$, then we say that $U$ is an ideal of $M$. An ideal $P$ of a $\Gamma$-ring 
$M$ is said to be prime if for any ideals $A$ and $B$ of $M, A \Gamma B \subseteq P$ implies $A \subseteq P$ or $B \subseteq P$. An ideal $P$ of a $\Gamma$ - ring $M$ is said to be semiprime if for any ideal $U$ of $M$, $U \Gamma U \subseteq P$ implies $U \subseteq P$. A $\Gamma$-ring $M$ is said to be semiprime if the zero ideal is semiprime. This definition is given as "A $\Gamma$-ring $M$ is said to be prime if $a \Gamma b=0$ with $a, b \in M$, implies $a=0$ or $b=0$ and semiprime $a \Gamma a=0$ with $a \in M$, implies $a=0$ " in [4, Theorem 2.2.23].

Let $\left(\Gamma_{1}, M_{1}\right)$ and $\left(\Gamma_{2}, M_{2}\right)$ be two gamma rings, $\phi: \Gamma_{1} \rightarrow \Gamma_{2}$ and $\theta: M_{1} \rightarrow M_{2}$ be two functions. Then an ordered pair $(\phi, \theta)$ of mappings is called a homomorphism of $\left(\Gamma_{1}, M_{1}\right)$ into $\left(\Gamma_{2}, M_{2}\right)$ if it satisfies the following properties:

(i) $\theta: M_{1} \rightarrow M_{2}$ is group homomorphism,

(ii) $\phi: \Gamma_{1} \rightarrow \Gamma_{2}$ is group homomorphism,

(iii) $\theta(x \alpha y)=\theta(x) \phi(\alpha) \theta(y)$, for all $x, y \in M, \alpha \in \Gamma$,

(iv) $\phi(\alpha x \beta)=\phi(\alpha) \theta(x) \phi(\beta)$, for all $x \in M, \alpha, \beta \in \Gamma$.

A homomorphism $(\phi, \theta)$ of a gamma ring $\left(\Gamma_{1}, M_{1}\right)$ into a gamma ring $\left(\Gamma_{2}, M_{2}\right)$ is called a monomorphism if $\phi$ and $\theta$ are one-one.

Let $M$ be a $\Gamma$-ring. A commutative additive group $N$ is called a right gamma $M$ module (or right $\Gamma M$-module) if for all $n, n_{1}, n_{2} \in N, m, m_{1}, m_{2} \in M$ and $\alpha, \beta \in \Gamma$,

(i) $n \alpha m \in N$,

(ii) $\left(n_{1}+n_{2}\right) \alpha m=n_{1} \alpha m+n_{2} \alpha m$,

(iii) $n(\alpha+\beta) m=n \alpha m+n \beta m$,

(iv) $n \alpha\left(m_{1}+m_{2}\right)=n \alpha m_{1}+n \alpha m_{2}$.

Let $N_{1}$ and $N_{2}$ be two right gamma $M$-modules. Then $\theta$ is called a right gamma $M$-module homomorphism ( or right $\Gamma M$-module homomorphism) of $N_{1}$ into $N_{2}$ if it satisfies the following properties:

(i) $\theta: N_{1} \rightarrow N_{2}$ is group homomorphism,

(ii) $\theta(x \alpha m)=\theta(x) \alpha m$, for all $x \in N_{1}, m \in M, \alpha \in \Gamma$.

A great deal work has been done on $\Gamma$-ring in the sense of Barnes and Nabusawa, the results can be compared to those of in the ring theory since 1964. On the other hand, it will be seen that rings of quotients play crucial role in the study of generalized identities in prime and semiprime rings. The study of two-sided rings of quotients was initiated by W. S. Martindale [6] for prime rings and extented for semiprime rings by S. A. Amitsur in [1]. The concept of centroid of a prime $\Gamma$-ring was defined and researched in [9], [8], [10] and [11]. In [4], it was defined the rings of quotients of a prime $\Gamma$-ring and researched the some properties of it. In this paper, we investigate the rings of quotients of a semiprime $\Gamma$-ring. It was first constructed by [12] for rings. We extend these results for semiprime $\Gamma$-rings.

Throughout the present paper, $M$ will a $\Gamma$-ring in the sense of Nabusawa and the symbol $(\Gamma, M)$ stands for the $(\Gamma, M)_{N}$. 


\section{RESULTS}

Definition 1. Let $M$ be a $\Gamma$-ring. If there exists $e \in M$ and $\delta \in \Gamma$ such that for all $x \in M e \delta x=x$, then $(\delta, e)$ is said to be strong left identity element of $(\Gamma, M)$. Similarly, if there exists $e \in M$ and $\delta \in \Gamma$ such that for all $x \in M x \delta e=x$, then $(\delta, e)$ is said to be strong right identity element of $(\Gamma, M)$. If $(\delta, e)$ is both a right and left strong identity element of $(\Gamma, M)$, then we say that $(\delta, e)$ is an strong identity element of $(\Gamma, M)$.

Definition 2. Let $M$ be a $\Gamma$-ring. A right ideal $J$ of $M$ is said to be dense if given any $0 \neq m_{1} \in M, m_{2} \in M$ there exists $m \in M, \gamma \in \Gamma$ such that $m_{1} \gamma m \neq 0$ and $m_{2} \gamma m \in J$. One defines a dense left ideal in an analogous fashion. The collection of all dense right ideal of $M$ will be denoted by $D(\Gamma, M)$.

Let $\mathrm{M}$ be a $\Gamma$-ring. For a subset $S$ of $M$,

$$
r_{\Gamma}(S)=\{c \in M \mid S \gamma c=(0), \forall \gamma \in \Gamma\}
$$

is called the right annihilator of $S$. A left annihilator $l_{\Gamma}(S)$ can be defined similarly.

Let $N$ be a $\Gamma M-$ module. For any submodule $J$ of $N$ and any subset $S \subseteq N$ we set

$$
(S: J)_{\Gamma}=\{c \in M \mid S \gamma c \subseteq J, \forall \gamma \in \Gamma\} .
$$

In particular, for any $a \in M$

$$
(a: J)_{\Gamma}=\{c \in M \mid a \gamma c \in J, \forall \gamma \in \Gamma\} .
$$

Theorem 1. Let $M$ be a semiprime $\Gamma$-ring, $U, V \in D(\Gamma, M)$ and $f: U \rightarrow M$ be a right $\Gamma M-$ module homomorphism. Then

i) $f^{-1}(V)=\{c \in U \mid f(c) \in V\} \in D(\Gamma, M)$.

ii) $(a: U)_{\Gamma} \in D(\Gamma, M)$ for all $a \in M$.

iii) $U \cap V \in D(\Gamma, M)$.

iv) If $W$ is a right ideal of $M$ and $U \subseteq W$, then $W \in D(\Gamma, M)$.

v) $l_{\Gamma}(U)=(0)=r_{\Gamma}(U)$.

vi) If $W$ is a right ideal of $M$ and $(a: W)_{\Gamma} \in D(\Gamma, M)$ for all $a \in M$, then $W \in$ $D(\Gamma, M)$.

vii) $U \Gamma V \in D(\Gamma, M)$.

Proof. i) Clearly, $f^{-1}(V)$ is a right ideal of $M$. Let $m_{1} \neq 0, m_{2} \in M$. By the dense right ideal $U$ of $M$, we get $m_{1} \gamma^{\prime} m^{\prime} \neq 0$ and $m_{2} \gamma^{\prime} m^{\prime} \in U$ for some $m^{\prime} \in M$, $\gamma^{\prime} \in \Gamma$. Since $f\left(m_{2} \gamma^{\prime} m^{\prime}\right) \in M$ and $V \in D(\Gamma, M)$, we see that there exists $m^{\prime \prime} \in M$, $\gamma^{\prime \prime} \in \Gamma$ such that $\left(m_{1} \gamma^{\prime} m^{\prime}\right) \gamma^{\prime \prime} m^{\prime \prime} \neq 0$ and $f\left(m_{2} \gamma^{\prime} m^{\prime}\right) \gamma^{\prime \prime} m^{\prime \prime} \in V$. Using $f$ is right $\Gamma M$-module homomorphism, we have

$$
f\left(m_{2} \gamma^{\prime} m^{\prime}\right) \gamma^{\prime \prime} m^{\prime \prime}=f\left(\left(m_{2} \gamma^{\prime} m^{\prime}\right) \gamma^{\prime \prime} m^{\prime \prime}\right)=f\left(m_{2} \gamma^{\prime}\left(m^{\prime} \gamma^{\prime \prime} m^{\prime \prime}\right)\right) .
$$

Setting $m=m^{\prime} \gamma^{\prime \prime} m^{\prime \prime}$, we conclude that $m_{1} \gamma^{\prime} m \neq 0$ and $f\left(m_{2} \gamma^{\prime} m\right) \in V$. That is, $m_{1} \gamma^{\prime} m \neq 0$ and $m_{2} \gamma^{\prime} m \in f^{-1}(V)$ for some $m \in M, \gamma^{\prime} \in \Gamma$. Thus, $f^{-1}(V) \in$ $D(\Gamma, M)$. 
ii) Consider the map $\lambda_{a \gamma}: M \rightarrow M, \lambda_{a \gamma}(m)=a \gamma m, \forall m \in M, \forall \gamma \in \Gamma$. One easily checks that $\lambda_{a \gamma}$ is right $\Gamma M$-module homomorphism. We have

$$
\begin{aligned}
\lambda_{a \gamma}^{-1}(U) & =\left\{c \in M \mid \lambda_{a \gamma}(c) \in U, \text { for all } \gamma \in \Gamma\right\} \\
& =\{c \in M \mid a \gamma c \in U, \text { for all } \gamma \in \Gamma\} \\
& =(a: U)_{\Gamma}
\end{aligned}
$$

According to (i), we find that $(a: U)_{\Gamma} \in D(\Gamma, M)$.

iii) If $i$ is the inclusion map $U \rightarrow M$, then

$$
i^{-1}(V)=U \cap V .
$$

Now apply $(i)$, we conclude that $U \cap V \in D(\Gamma, M)$.

iv) Let $W$ be a right ideal of $M, U \subseteq W$ and $m_{1} \neq 0, m_{2} \in M$. Since $U$ is a dense right ideal of $M$, we obtain that $m_{1} \gamma m \neq 0$ and $m_{2} \gamma m \in U$ for some $m \in M, \gamma \in \Gamma$. Using $U \subseteq W$, we get $m_{1} \gamma m \neq 0$ and $m_{2} \gamma m \in W$. That is $W \in D(\Gamma, M)$.

v) We assume that $U \gamma c=(0)$ for some $0 \neq c \in M$, for all $\gamma \in \Gamma$. Setting $m_{1}=$ $c=m_{2}$, we arrive that there exists $\alpha \in \Gamma, m \in M$ such that $0 \neq c \alpha m \in U$. Hence

$$
(c \alpha m) \gamma(c \alpha m) \in(U \gamma c) \alpha m=(0) \text { for all } \gamma \in \Gamma \text {, }
$$

and so

$$
(c \alpha m) \Gamma(c \alpha m)=(0) .
$$

By the semiprimeness of $(\Gamma, M)$, we have $c \alpha m=0$. It contradicts $c \alpha m \neq 0$. Hence $r_{\Gamma}(U)=(0)$.

Next we suppose $l_{\Gamma}(U) \neq(0)$. We see that there exists $0 \neq c \in M$ such that $c \gamma U=(0)$ for all $\gamma \in \Gamma$. On the other hand, $c \alpha m \neq 0$ for some $\alpha \in \Gamma, m \in M$. Indeed, if $c \alpha m=0$ for all $\alpha \in \Gamma, m \in M$, then $c \Gamma c=(0)$, and so $c=0$ by the semiprimeness of $(\Gamma, M)$. But we know that $c \neq 0$.

Setting $m_{1}=c \alpha m \neq 0, m_{2}=m$, we have $(c \alpha m) \gamma^{\prime} m^{\prime} \neq 0$ and $m \gamma^{\prime} m^{\prime} \in U$ for some $m^{\prime} \in M, \gamma^{\prime} \in \Gamma$. But

$$
(c \alpha m) \gamma^{\prime} m^{\prime}=c \alpha\left(m \gamma^{\prime} m^{\prime}\right) \in c \Gamma U=(0)
$$

and a contradiction is reached. So we must have $l_{\Gamma}(U)=(0)$.

vi) Let $m_{1} \neq 0, m_{2} \in M$. Using $U \in D(\Gamma, M)$, we see that there exists $m^{\prime} \in$ $M, \gamma^{\prime} \in \Gamma$ such that $m_{1} \gamma^{\prime} m^{\prime} \neq 0$ and $m_{2} \gamma^{\prime} m^{\prime} \in U$. By the hypothesis, we have $\left(m_{2} \gamma^{\prime} m^{\prime}: W\right)_{\Gamma} \in D(\Gamma, M)$. By (v), we get $l_{\Gamma}\left(\left(m_{2} \gamma^{\prime} m^{\prime}: W\right)_{\Gamma}\right)=(0)$. Hence $m_{1} \gamma^{\prime} m^{\prime} \neq 0$, we obtain $m_{1} \gamma^{\prime} m^{\prime} \notin l_{\Gamma}\left(\left(m_{2} \gamma^{\prime} m^{\prime}: W\right)_{\Gamma}\right)$. That is $\left(m_{1} \gamma^{\prime} m^{\prime}\right) \gamma^{\prime \prime} m^{\prime \prime} \neq 0$ for some $m^{\prime \prime} \in\left(m_{2} \gamma^{\prime} m^{\prime}: W\right)_{\Gamma}, \gamma^{\prime \prime} \in \Gamma$. Therefore,

$$
\left(m_{1} \gamma^{\prime} m^{\prime}\right) \gamma^{\prime \prime} m^{\prime \prime}=m_{1} \gamma^{\prime}\left(m^{\prime} \gamma^{\prime \prime} m^{\prime \prime}\right) \neq 0
$$

and $\left(m_{2} \gamma^{\prime} m^{\prime}\right) \gamma^{\prime \prime} m^{\prime \prime}=m_{2} \gamma^{\prime}\left(m^{\prime} \gamma^{\prime \prime} m^{\prime \prime}\right) \in W$.

Setting $m=m^{\prime} \gamma^{\prime \prime} m^{\prime \prime}$, we conclude that there exists $m \in M, \gamma^{\prime} \in \Gamma$ such that $m_{1} \gamma^{\prime} m \neq 0$ and $m_{2} \gamma^{\prime} m \in W$. Thus $W$ is a dense right ideal of $M$. 
vii) Let $m_{1} \neq 0, m_{2} \in M$. By (ii), we have $\left(m_{2}: U\right)_{\Gamma} \in D(\Gamma, M)$. Using (v), we get $l_{\Gamma}\left(\left(m_{2}: U\right)_{\Gamma}\right)=(0)$. We obtain $m_{1} \notin l_{\Gamma}\left(\left(m_{2}: U\right)_{\Gamma}\right)$. Then we have $m_{1} \gamma^{\prime} m^{\prime} \neq$ 0 for some $m^{\prime} \in\left(m_{2}: U\right)_{\Gamma}, \gamma^{\prime} \in \Gamma$. On the other hand, we see that there exists $m^{\prime \prime} \in V, \gamma^{\prime \prime} \in \Gamma$ such that $\left(m_{1} \gamma^{\prime} m^{\prime}\right) \gamma^{\prime \prime} m^{\prime \prime} \neq 0$. Indeed, if $\left(m_{1} \gamma^{\prime} m^{\prime}\right) \gamma^{\prime \prime} m^{\prime \prime}=0$, for all $m^{\prime \prime} \in V, \gamma^{\prime \prime} \in \Gamma$, then $m_{1} \gamma^{\prime} m^{\prime} \in l_{\Gamma}(V)$. Also, we know that $l_{\Gamma}(V)=(0)$. Hence we get $m_{1} \gamma^{\prime} m^{\prime}=0$. This is a contradiction.

Setting $m=m^{\prime} \gamma^{\prime \prime} m^{\prime \prime}$, we obtain that $m_{1} \gamma^{\prime} m \neq 0$ and $m_{2} \gamma^{\prime} m=m_{2} \gamma^{\prime}\left(m^{\prime} \gamma^{\prime \prime} m^{\prime \prime}\right)=\left(m_{2} \gamma^{\prime} m^{\prime}\right) \gamma^{\prime \prime} m^{\prime \prime} \in U \Gamma V$. Therefore, $U \Gamma V \in D(\Gamma, M)$.

Corollary 1. Let $M$ be a semiprime $\Gamma$-ring and $U$ be a right ideal of $M$. Then $U \in D(\Gamma, M)$ if and only if $l_{\Gamma}\left((a: U)_{\Gamma}\right)=(0)$ for all $a \in M$.

Proof. If $U \in D(\Gamma, M)$, then $(a: U)_{\Gamma} \in D(\Gamma, M)$ for all $a \in M$ by Theorem 1(ii). By Theorem 1(v), we have $l_{\Gamma}\left((a: U)_{\Gamma}\right)=(0)$ for all $a \in M$. Conversely, let $m_{1} \neq 0, m_{2} \in M$. Since $m_{1} \neq 0$, we get $m_{1} \notin l_{\Gamma}\left(\left(m_{2}: U\right)_{\Gamma}\right)$. Thus, $m_{1} \gamma m \neq 0$ for some $m \in\left(m_{2}: U\right)_{\Gamma}, \gamma \in \Gamma$. That is, $m_{1} \gamma m \neq 0$ and $m_{2} \gamma m \in U$ for some $m \in M$, $\gamma \in \Gamma$. We get $U \in D(\Gamma, M)$. This completes the proof.

We are now in a position to construct the desired gamma ring of quotients of $M$. Let $M$ be a semiprime $\Gamma$-ring. Consider the set

$$
\aleph=\{(f ; U) \mid U \in D(\Gamma, M)\}
$$

\section{$f: U \rightarrow M$ is a right $\Gamma M$-module homomorphism}

We define $(f ; U) \sim(g ; V)$ if there exists $W \subseteq U \cap V$ such that $W \in D(\Gamma, M)$ and $f=g$ on $W$. By [3], one easily checks that " $\sim$ " is an equivalence relation and we let $[f ; U]$ denote the equivalence class determined by $(f ; U) \in \aleph$. Let $Q$ be the set of all equivalence classes. We define addition on $Q$ as follow:

$$
[f ; U]+[g ; V]=[f+g ; U \cap V]
$$

First of all we note that by Theorem 1(iii), $U \cap V \in D(\Gamma, M)$. Using similar arguments as in [3], we can prove that $Q$ is a abelian additive group.

In a similar fasion, let $(M, \Gamma)$ be a semiprime gamma ring. The collection of all dense left ideal of $(M, \Gamma)$ will be denoted by $D(M, \Gamma)$.

$\Re=\{(\tau ; \Omega) \mid \Omega \in D(M, \Gamma), \tau: \Omega \rightarrow \Gamma$ is a left $M \Gamma$-module homomorphism $\}$

We will show that $\Gamma$ is a left $M \Gamma$-module. Indeed, since $(M, \Gamma)$ is a gamma ring, $\Gamma$ is abelian additive group. Also, for all $\gamma, \gamma_{1}, \gamma_{2} \in \Gamma, m, m_{1}, m_{2} \in M$ and $\alpha, \alpha_{1}, \alpha_{2} \in \Gamma$,

(i) $\alpha m \gamma \in \Gamma$,

(ii) $\alpha m\left(\gamma_{1}+\gamma_{2}\right)=\alpha m \gamma_{1}+\alpha m \gamma_{2}$

(iii) $\alpha\left(m_{1}+m_{2}\right) \gamma=\alpha m_{1} \gamma+\alpha m_{2} \gamma$,

(iv) $\left(\alpha_{1}+\alpha_{2}\right) m \gamma=\alpha_{1} m \gamma+\alpha_{2} m \gamma$.

On the other hand, $\Omega$ is selected to be left $M \Gamma$-module. 
We define $(\tau ; \Omega) \approx(\sigma ; \Lambda)$ if there exists $\Pi \subseteq \Omega \cap \Lambda$ such that $\Pi \in D(M, \Gamma)$ and $\tau=\sigma$ on $\Pi$. " $\approx$ " is an equivalence relation. Let $[\tau ; \Omega]$ denote the equivalence class determined by $(\tau ; \Omega) \in \Re$ and $\Delta$ denote the set of all equivalence classes. We define addition of equivalence classes as follow:

$$
[\tau ; \Omega]+[\sigma ; \Lambda]=[\tau+\sigma ; \Omega \cap \Lambda]
$$

In similar fasion, one easily checks that $\Delta$ is abelian additive group.

Let $\tau: \Omega \rightarrow \Gamma$ be a left $M \Gamma$-module homomorphism. Define

$$
\stackrel{\Delta}{\tau}: M \Omega M \rightarrow M \text { by } \stackrel{\Delta}{\tau}(m \gamma n)=m \tau(\gamma) n \text { for all } m, n \in M, \gamma \in \Omega .
$$

Now we define multiplication of equivalence classes as follow:

$$
[f ; U][\tau ; \Omega][g ; V]=\left[f^{\Delta} g ;(\tilde{\tau} g)^{-1}(U)\right]
$$

where $[f ; U],[g ; V] \in Q$ and $[\tau ; \Omega] \in \Delta$. We will show that multiplication is well defined. For any $\left[f_{1} ; U_{1}\right],\left[f_{2} ; U_{2}\right],\left[g_{1} ; V_{1}\right],\left[g_{2} ; V_{2}\right] \in Q$ and $\left[\tau_{1} ; \Omega_{1}\right],\left[\tau_{2} ; \Omega_{2}\right] \in \Delta$, we get

$$
\left(\left[f_{1} ; U_{1}\right],\left[\tau_{1} ; \Omega_{1}\right],\left[g_{1} ; V_{1}\right]\right)=\left(\left[f_{2} ; U_{2}\right],\left[\tau_{2} ; \Omega_{2}\right],\left[g_{2} ; V_{2}\right]\right)
$$

Then

$$
\left[f_{1} ; U_{1}\right]=\left[f_{2} ; U_{2}\right],\left[\tau_{1} ; \Omega_{1}\right]=\left[\tau_{2} ; \Omega_{2}\right],\left[g_{1} ; V_{1}\right]=\left[g_{2} ; V_{2}\right],
$$

and so

$$
\left(f_{1} ; U_{1}\right) \sim\left(f_{2} ; U_{2}\right),\left(\tau_{1} ; \Omega_{1}\right) \approx\left(\tau_{2} ; \Omega_{2}\right),\left(g_{1} ; V_{1}\right) \sim\left(g_{2} ; V_{2}\right) .
$$

Hence, there exists $W_{1}, W_{2} \in D(\Gamma, M)$ such that $W_{1} \subseteq U_{1} \cap U_{2}, W_{2} \subseteq V_{1} \cap V_{2}$, $f_{1}=f_{2}$ on $W_{1}, g_{1}=g_{2}$ on $W_{2}$ and $\Pi \in D(M, \Gamma)$ such that $\Pi \subseteq \Omega_{1} \cap \Omega_{2}, \tau_{1}=\tau_{2}$ on $\Pi$.

Set $W=g_{1}^{-1}\left(W_{1} \Pi M\right) \cap W_{2}$. Using the same techniques in Theorem 1(vii), we prove that $W_{1} \Pi M \in D(\Gamma, M)$. By Theorem 1(i), we have $g_{1}^{-1}\left(W_{1} \Pi M\right) \in$ $D(\Gamma, M)$. Also $g_{1}^{-1}\left(W_{1} \Pi M\right) \cap W_{2} \in D(\Gamma, M)$ by Theorem 1(iii).

Now, we will show that $W \subseteq\left(\stackrel{\Delta}{\tau_{1}} g_{1}\right)^{-1}\left(U_{1}\right) \cap\left(\frac{\Delta}{\tau_{2}} g_{2}\right)^{-1}\left(U_{2}\right)$. Let $x$ be any element of $W=g_{1}^{-1}\left(W_{1} \Pi M\right) \cap W_{2}$. That is, $g_{1}(x) \in W_{1} \Pi M$ and $x \in W_{2}$. Taking $g_{1}(x)$ by $w_{1} \gamma m$, where $w_{1} \in W_{1}, \gamma \in \Pi, m \in M$, we get

$$
\stackrel{\Delta}{\tau}_{1} g_{1}(x)=\stackrel{\Delta}{\tau_{1}}\left(g_{1}(x)\right)=\stackrel{\Delta}{\tau_{1}}\left(w_{1} \gamma m\right)=w_{1} \tau_{1}(\gamma) m .
$$

On the other hand, $g_{1}(x)=g_{2}(x)$ for $x \in W_{2}$, there by obtain

$$
\stackrel{\Delta}{\tau_{2}} g_{2}(x)=\stackrel{\Delta}{\tau_{2}}\left(g_{2}(x)\right)=\stackrel{\Delta}{\tau_{2}}\left(g_{1}(x)\right)=\stackrel{\Delta}{\tau_{2}}\left(w_{1} \gamma m\right)=w_{1} \tau_{2}(\gamma) m .
$$

For $w_{1} \in W_{1} \subseteq U_{1} \cap U_{2}$, we conclude that

$$
\stackrel{\Delta}{\tau_{1}} g_{1}(x)=w_{1} \tau_{1}(\gamma) m \in U_{1} \text { and } \stackrel{\Delta}{\tau_{2}} g_{2}(x)=w_{1} \tau_{2}(\gamma) m \in U_{2} .
$$


Hence, we find that $x \in\left(\frac{\Delta}{\tau_{1}} g_{1}\right)^{-1}\left(U_{1}\right)$ and $x \in\left(\frac{\Delta}{\tau_{2}} g_{2}\right)^{-1}\left(U_{2}\right)$, i.e., $x \in\left(\frac{\Delta}{\tau_{1}} g_{1}\right)^{-1}\left(U_{1}\right) \cap$ $\left(\hat{\tau}_{2} g_{2}\right)^{-1}\left(U_{2}\right)$.

Moreover, we prove that $f_{1} \hat{\tau}_{1} g_{1}=f_{2} \stackrel{\Delta}{\tau_{2}} g_{2}$ on $W$. Let $x \in W$. We know that $g_{1}(x) \in W_{1} \Pi M$. Replacing $g_{1}(x)$ by $w_{1} \gamma m$, where $w_{1} \in W_{1}, \gamma \in \Pi, m \in M$, we have

$$
\left(f_{1} \stackrel{\Delta}{\tau_{1}} g_{1}\right)(x)=f_{1}\left(\hat{\tau}_{1}\left(g_{1}(x)\right)\right)=f_{1}\left(\Delta_{1}\left(w_{1} \gamma m\right)\right)=f_{1}\left(w_{1} \tau_{1}(\gamma) m\right) .
$$

Using $w_{1} \in W_{1} \subseteq U_{1}$ and $f_{1}$ is a right $\Gamma M$-module homomorphism, we get

$$
f_{1}\left(w_{1} \tau_{1}(\gamma) m\right)=f_{1}\left(w_{1}\right) \tau_{1}(\gamma) m .
$$

Since $f_{1}=f_{2}$ on $W_{1}$ and $\tau_{1}=\tau_{2}$ on $\Pi$, we obtain that

$$
f_{1}\left(w_{1}\right) \tau_{1}(\gamma) m=f_{2}\left(w_{1}\right) \tau_{2}(\gamma) m=f_{2}\left(w_{1} \tau_{2}(\gamma) m\right)=f_{2}\left(\tau_{2}\left(w_{1} \gamma m\right)\right) .
$$

Appliying $x \in W_{2}$ and $g_{1}=g_{2}$ on $W_{2}$, we conclude that

$$
\left(f_{2} \stackrel{\Delta}{\tau_{2}}\right)\left(w_{1} \gamma m\right)=f_{2}\left(\stackrel{\Delta}{\tau_{2}}\left(g_{1}(x)\right)\right)=\left(f_{2} \stackrel{\Delta}{\tau_{2}} g_{2}\right)(x) .
$$

Hence we find that $f_{1} \Delta_{1} g_{1}=f_{2}{ }_{2} g_{2}$ on $W$, and also

Therefore,

$$
\left[f_{1} \tau_{1} g_{1} ;\left(\tilde{\tau}_{1} g_{1}\right)^{-1}\left(U_{1}\right)\right]=\left[f_{2} \stackrel{\Delta}{\tau_{2}} g_{2} ;\left(\tilde{\tau}_{2} g_{2}\right)^{-1}\left(U_{2}\right)\right] .
$$

As a result multiplication is well defined. Also, for all $[f ; U],[g ; V] \in Q$ and $[\tau ; \Omega] \in$ $\Delta$, we get $[f ; U][\tau ; \Omega][g ; V] \in Q$. Indeed, we defined that $[f ; U][\tau ; \Omega][g ; V]=\left[f \frac{\Delta}{\tau} g ;\left(\frac{\Delta}{\tau} g\right)^{-1}(U)\right]$ by Theorem 1(i), we have $(\stackrel{\Delta}{\tau} g)^{-1}(U) \in$ $D(\Gamma, M)$. It is clear that $f^{\Delta} \mathrm{\tau} g$ is a right $\Gamma M$-module homomorphism. Thus, $[f ; U][\tau ; \Omega][g ; V] \in Q$ for all $[f ; U],[g ; V] \in Q$ and $[\tau ; \Omega] \in \Delta$.

Now we will show that $(\Delta, Q)$ is a gamma ring.

a) i) For all $[f ; U],[g ; V],[h ; K] \in Q$ and $[\tau ; \Omega] \in \Delta$,

$$
\begin{aligned}
& ([f ; U]+[g ; V])[\tau ; \Omega][h ; K]=[f+g ; U \cap V][\tau ; \Omega][h ; K] \\
& =\left[(f+g) \stackrel{\Delta}{\tau} h ;\left(\frac{\Delta}{\tau} h\right)^{-1}(U \cap V)\right] \\
& =\left[f^{\Delta} \frac{\Delta}{\tau} h+g \stackrel{\Delta}{\tau} h ;(\tilde{\tau} h)^{-1}(U) \cap\left(\frac{\Delta}{\tau} h\right)^{-1}(V)\right] \\
& =\left[f^{\Delta} \stackrel{\Delta}{\tau} h ;(\stackrel{\Delta}{\tau} h)^{-1}(U)\right]+\left[g \stackrel{\Delta}{\tau} h ;(\stackrel{\Delta}{\tau} h)^{-1}(V)\right] \\
& =[f ; U][\tau ; \Omega][h ; K]+[g ; V][\tau ; \Omega][h ; K] .
\end{aligned}
$$


ii) For all $[f ; U],[g ; V] \in Q$ and $[\tau ; \Omega],[\sigma ; \Lambda] \in \Delta$,

$$
\begin{aligned}
{[f ; U]([\tau ; \Omega]+[\sigma ; \Lambda])[g ; V] } & =[f ; U][\tau+\sigma ; \Omega \cap \Lambda][g ; V] \\
& =\left[f \left(\begin{array}{c}
\Delta \\
\end{array}\left(\begin{array}{c}
\Delta \\
\Delta
\end{array} ;((\tau+\sigma) g)^{-1}(U)\right],\right.\right.
\end{aligned}
$$

and

$$
\begin{aligned}
& {[f ; U][\tau ; \Omega][g ; V]+[f ; U][\sigma ; \Lambda][g ; V]} \\
& =\left[f^{\Delta} g^{\prime} ;(\dot{\Delta} g)^{-1}(U)\right]+\left[f \stackrel{\Delta}{\sigma} g ;(\Delta g)^{-1}(U)\right] \\
& =\left[f^{\Delta} \tilde{\tau} g+f^{\Delta} \stackrel{\Delta}{\sigma} ;(\stackrel{\Delta}{\tau} g)^{-1}(U) \cap(\stackrel{\Delta}{\sigma} g)^{-1}(U)\right] \text {. }
\end{aligned}
$$

We will show that (2.1) and (2.3) are equivalent. Let $W=(\stackrel{\Delta}{\tau} g)^{-1}(U) \cap(\stackrel{\Delta}{\sigma} g)^{-1}(U)$ and $x \in W$. Then $x \in(\stackrel{\Delta}{\tau} g)^{-1}(U)$ and $x \in(\stackrel{\Delta}{\sigma} g)^{-1}(U)$, i.e., $(\stackrel{\Delta}{\tau} g)(x) \in U$ and $(\dot{\sigma} g)(x) \in U$. Thus $(\stackrel{\Delta}{\tau} g)(x)+(\vec{\sigma} g)(x) \in U$ and so $(\stackrel{\Delta}{\tau}+\stackrel{\Delta}{\sigma}) g(x) \in U$. That is, $x \in((\tau+\sigma) g)^{-1}(U)$. Hence

$$
W \subseteq((\tau+\sigma) g)^{-1}(U) \cap(\stackrel{\Delta}{\tau} g)^{-1}(U) \cap(\stackrel{\Delta}{\sigma} g)^{-1}(U) .
$$

Moreover, for all $w \in W$

$$
(f(\tau+\stackrel{\Delta}{\tau}) g)(w)=\left(f^{\Delta} \frac{\Delta}{\tau}\right)(w)+(f \stackrel{\Delta}{\sigma} g)(w)=\left(f^{\Delta} \frac{\Delta}{\tau} g+f \stackrel{\Delta}{\sigma} g\right)(w) .
$$

iii) For all $[f ; U],[g ; V],[h ; K] \in Q$ and $[\tau ; \Omega] \in \Delta$,

$$
\begin{aligned}
& {[f ; U][\tau ; \Omega]([g ; V]+[h ; K])=[f ; U][\tau ; \Omega][g+h ; V \cap K]} \\
& =\left[f^{\Delta}(g+h) ;(\tilde{\tau}(g+h))^{-1}(U)\right] \\
& =\left[f^{\Delta} \frac{\Delta}{\tau} g+f^{\Delta} \tau h ;(\tilde{\tau} g)^{-1}(U) \cap(\stackrel{\Delta}{\tau} h)^{-1}(U)\right] \\
& =\left[f^{\Delta} \underset{\tau}{g} ;(\stackrel{\Delta}{\tau} g)^{-1}(U)\right]+\left[f^{\Delta} h ;(\stackrel{\Delta}{\tau} h)^{-1}(U)\right] \\
& =[f ; U][\tau ; \Omega][g ; V]+[f ; U][\tau ; \Omega][h ; K] \text {. }
\end{aligned}
$$

Selected $W=(\stackrel{\Delta}{\tau} g)^{-1}(U) \cap\left(\hat{\tau}_{h}\right)^{-1}(U)$, above equality is proved in anagous way. b) i) For all $[f ; U],[g ; V],[h ; K] \in Q$ and $[\tau ; \Omega],[\sigma ; \Lambda] \in \Delta$,

$$
([f ; U][\tau ; \Omega][g ; V])[\sigma ; \Lambda][h ; K]=\left[f^{\Delta} \stackrel{\Delta}{\tau} ;(\stackrel{\Delta}{\tau} g)^{-1}(U)\right][\sigma ; \Lambda][h ; K]
$$




$$
\begin{aligned}
& =\left[\left(f^{\stackrel{\Delta}{\tau}} g\right) \stackrel{\Delta}{\sigma} h ;(\stackrel{\Delta}{\sigma} h)^{-1}(\stackrel{\Delta}{\tau} g)^{-1}(U)\right] . \\
{[f ; U][\tau ; \Omega]([g ; V][\sigma ; \Lambda][h ; K]) } & =[f ; U][\tau ; \Omega]\left[g \stackrel{\Delta}{\sigma} h ;(\stackrel{\Delta}{\sigma} h)^{-1}(V)\right] \\
& =\left[f^{\Delta} \stackrel{\Delta}{\tau}(g \stackrel{\Delta}{\sigma} h) ;(\stackrel{\Delta}{\tau}(g \stackrel{\Delta}{\sigma} h))^{-1}(U)\right]
\end{aligned}
$$

We will prove that (2.4) and (2.5) are equivalent. Let $W=(\stackrel{\Delta}{\sigma} h)^{-1}(\stackrel{\Delta}{\tau} g)^{-1}(U)$ and $x \in W$. Then $(\stackrel{\Delta}{\sigma} h)(x) \in\left(\frac{\Delta}{\tau} g\right)^{-1}(U)$ and so $\left(\frac{\Delta}{\tau} g\right)(\stackrel{\Delta}{\sigma} h)(x) \in U$. Finally, $x \in$ $(\stackrel{\Delta}{\tau}(g \stackrel{\Delta}{\sigma} h))^{-1}(U)$. Thus $W \subseteq(\stackrel{\Delta}{\sigma} h)^{-1}(\stackrel{\Delta}{\tau} g)^{-1}(U) \cap(\stackrel{\Delta}{\tau}(g \stackrel{\Delta}{\sigma} h))^{-1}(U)$, and also

$$
\left(\left(f^{\stackrel{\Delta}{\tau} g}\right) \stackrel{\Delta}{\sigma} h\right)(w)=\left(f^{\Delta} \underset{\sim}{g}(\stackrel{\Delta}{\sigma} h)\right)(w), \text { for all } w \in W .
$$

ii) For all $[f ; U],[g ; V],[h ; K] \in Q$ and $[\tau ; \Omega],[\sigma ; \Lambda] \in \Delta$,

$$
\begin{aligned}
& ([f ; U][\tau ; \Omega][g ; V])[\sigma ; \Lambda][h ; K]=\left[f^{\Delta} \tau g ;(\tau g)^{-1}(U)\right][\sigma ; \Lambda][h ; K] \\
& =\left[\left(f^{\Delta} \underset{\tau}{g}\right) \stackrel{\Delta}{\sigma} h ;(\stackrel{\Delta}{\sigma} h)^{-1}(\stackrel{\Delta}{\tau} g)^{-1}(U)\right] \\
& =\left[f(\tilde{\tau} g \sigma) h ;(\tilde{\tau} g \sigma \sigma h)^{-1}(U)\right] \\
& =[f ; U]\left[\stackrel{\Delta}{\tau} g \Delta ; \dot{\sigma} ;(g \sigma)^{-1}(\Omega)\right][h ; K] \\
& =[f ; U]([\tau ; \Omega][g ; V][\sigma ; \Lambda])[h ; K] \text {. }
\end{aligned}
$$

Similar to the above can be shown.

c) Let $[f ; U][\tau ; \Omega][g ; V]=[0 ; M]$ for all $[f ; U],[g ; V] \in Q$ and $[\tau ; \Omega] \in \Delta$. For $\left[I_{M} ; M\right] \in Q$, where $I_{M}: M \rightarrow M$, is an identity right $\Gamma M$-module homomorphism, we get

$$
\left[I_{M} ; M\right][\tau ; \Omega]\left[I_{M} ; M\right]=[0 ; M],
$$

and so

$$
\left[I_{M} \stackrel{\Delta}{\tau} I_{M} ;\left(\stackrel{\Delta}{\tau} I_{M}\right)^{-1}(M)\right]=[0 ; M] .
$$

Let $W=M \Omega M$. We first prove that $M \Omega M \subseteq M \cap\left(\stackrel{\Delta}{\tau} I_{M}\right)^{-1}(M)$. For any $x=$ $m \gamma n \in M \Omega M$, where $m, n \in M, \gamma \in \Omega$, we have

$$
\left(\stackrel{\Delta}{\tau} I_{M}\right)(x)=\left(\stackrel{\Delta}{\tau} I_{M}\right)(m \gamma n)=\stackrel{\Delta}{\tau}\left(I_{M}(m \gamma n)\right)=\stackrel{\Delta}{\tau}(m \gamma n)=m \tau(\gamma) n .
$$

Thus we get $\left(\stackrel{\Delta}{\tau} I_{M}\right)(x) \in M$, and so $x \in\left({ }_{\tau} I_{M}\right)^{-1}(M)$. On the other hand, we know that $x \in M$. Hence $x \in M \cap\left(\stackrel{\Delta}{\tau} I_{M}\right)^{-1}(M)$. 
Now, for all $w=m \gamma n$, where $m, n \in M, \gamma \in \Omega$,

$$
I_{M} \stackrel{\Delta}{\tau} I_{M}(w)=I_{M} \stackrel{\Delta}{\tau} I_{M}(m \gamma n)=0(m \gamma n) .
$$

That is

$$
m \tau(\gamma) n=0, \text { for all } m, n \in M \text { and } \gamma \in \Omega .
$$

Since $(\Gamma, M)$ is gamma ring, we have $\tau(\gamma)=0$, for all $\gamma \in \Omega$, and so, $\tau=0$. That is, $[\tau ; \Omega]=[0 ; \Gamma]$.

So, we see that $(\Delta, Q)$ is a gamma ring and we call it the maximal right gamma ring of quotients of $(\Gamma, M)$.

Theorem 2. Let $(M, \Gamma)$ be a semiprime gamma ring. The collection of all dense left ideal of $(M, \Gamma)$ will be denoted by $D(M, \Gamma), \Omega, \Lambda \in D(M, \Gamma)$ and $\tau: \Omega \rightarrow \Gamma$ be a left $M \Gamma$-module homomorphism. Then

i) $\tau^{-1}(\Lambda)=\{\gamma \in \Omega \mid \tau(\gamma) \in \Lambda\} \in D(M, \Gamma)$.

ii) $(\alpha: \Omega)_{M} \in D(M, \Gamma)$ for all $\alpha \in \Gamma$.

iii) $\Omega \cap \Lambda \in D(M, \Gamma)$.

iv) If $\Pi$ is a left ideal of $\Gamma$ and $\Omega \subseteq \Pi$, then $\Pi \in D(M, \Gamma)$.

v) $l_{M}(\Omega)=(0)=r_{M}(\Omega)$.

vi) If $\Pi$ is a left ideal of $\Gamma$ and $(\alpha: \Pi)_{M} \in D(M, \Gamma)$ for all $\alpha \in \Gamma$, then $\Pi \in$ $D(M, \Gamma)$.

vii) $\Omega M \Lambda \in D(M, \Gamma)$.

Proof. The procedures in Theorem 1 can be exactly applied in set $D(M, \Gamma)$ and the same results are obtained.

One can construct the maximal left gamma ring of quotient of $(M, \Gamma)$ using the following operations. Now we can define multiplication of equivalence classes as follow:

$$
[\tau ; \Omega][f ; U][\sigma ; \Lambda]=\left[\begin{array}{c}
\Delta \\
\tau f \sigma ;(f \sigma)^{-1}(\Omega)
\end{array}\right]
$$

where $\stackrel{\Delta}{f}: \Gamma U \Gamma \rightarrow \Gamma \stackrel{\Delta}{f}(\gamma m \beta)=\gamma f(m) \beta$ and $[f ; U] \in Q$ and $[\tau ; \Omega],[\sigma ; \Lambda] \in \Delta$.

We will prove that multiplication is well defined. For any $\left[\tau_{1} ; \Omega_{1}\right],\left[\tau_{2} ; \Omega_{2}\right],\left[\sigma_{1} ; \Lambda_{1}\right],\left[\sigma_{2} ; \Lambda_{2}\right] \in \Delta$ and $\left[f_{1} ; U_{1}\right],\left[f_{2} ; U_{2}\right] \in Q$, we get

$$
\left(\left[\tau_{1} ; \Omega_{1}\right],\left[f_{1} ; U_{1}\right],\left[\sigma_{1} ; \Lambda_{1}\right]\right)=\left(\left[\tau_{2} ; \Omega_{2}\right],\left[f_{2} ; U_{2}\right],\left[\sigma_{2} ; \Lambda_{2}\right]\right) .
$$

Then

$$
\left[\tau_{1} ; \Omega_{1}\right]=\left[\tau_{2} ; \Omega_{2}\right],\left[f_{1} ; U_{1}\right]=\left[f_{2} ; U_{2}\right],\left[\sigma_{1} ; \Lambda_{1}\right],\left[\sigma_{2} ; \Lambda_{2}\right]
$$

and so

$$
\left(\tau_{1} ; \Omega_{1}\right) \approx\left(\tau_{2} ; \Omega_{2}\right),\left(f_{1} ; U_{1}\right) \sim\left(f_{2} ; U_{2}\right),\left(\sigma_{1} ; \Lambda_{1}\right) \approx\left(\sigma_{2} ; \Lambda_{2}\right) .
$$


Therefore, there exists $\Pi_{1}, \Pi_{2} \in D(M, \Gamma)$ such that $\Pi_{1} \subseteq \Omega_{1} \cap \Omega_{2}, \Pi_{2} \subseteq \Lambda_{1} \cap$ $\Lambda_{2}, \tau_{1}=\tau_{2}$ on $\Pi_{1}, \sigma_{1}=\sigma_{2}$ on $\Pi_{2}$ and $W \in D(\Gamma, M)$ such that $W \subseteq U_{1} \cap U_{2}$, $f_{1}=f_{2}$ on $W$.

Let $\Pi=\sigma_{1}^{-1}\left(\Gamma W \Pi_{1}\right) \cap \Pi_{2}$. Using Theorem 2(vii), we show that $\Gamma W \Pi_{1} \in$ $D(M, \Gamma)$. By Theorem 2(i), we see that $\sigma_{1}^{-1}\left(\Gamma W \Pi_{1}\right) \in D(M, \Gamma)$. Hence $\sigma_{1}^{-1}\left(\Gamma W \Pi_{1}\right) \cap \Pi_{2} \in D(M, \Gamma)$ by Theorem 2(iii).

Now, we will show that $\Pi \subseteq\left(\stackrel{\Delta}{\left.f_{1} \sigma_{1}\right)^{-1}}\left(\Omega_{1}\right) \cap\left(\stackrel{\Delta}{f_{2} \sigma_{2}}\right)^{-1}\left(\Omega_{2}\right)\right.$. Let $\alpha$ be any element of $\Pi=\sigma_{1}^{-1}\left(\Gamma W \Pi_{1}\right) \cap \Pi_{2}$. That is $\sigma_{1}(\alpha) \in \Gamma W \Pi_{1}$ and $\alpha \in \Pi_{2}$. Replacing $\sigma_{1}(\alpha)$ by $\gamma w \beta_{1}$, where $\beta_{1} \in \Pi_{1}, w \in W, \gamma \in \Gamma$, yields that

$$
\left(\stackrel{\Delta}{f_{1} \sigma_{1}}\right)(\alpha)=\stackrel{\Delta}{f_{1}}\left(\sigma_{1}(\alpha)\right)=\stackrel{\Delta}{f_{1}}\left(\gamma w \beta_{1}\right)=\gamma f_{1}(w) \beta_{1} .
$$

On the other hand, $\sigma_{1}(\alpha)=\sigma_{2}(\alpha)$ for $\alpha \in \Pi_{2}$, we get

$$
\stackrel{\Delta}{\left(f_{2} \sigma_{2}\right)}(\alpha)=\stackrel{\Delta}{f_{2}}\left(\sigma_{2}(\alpha)\right)=\stackrel{\Delta}{f_{2}}\left(\sigma_{1}(\alpha)\right)=\stackrel{\Delta}{f_{2}}\left(\gamma w \beta_{1}\right)=\gamma f_{2}(w) \beta_{1} .
$$

For $\beta_{1} \in \Pi_{1} \subseteq \Omega_{1} \cap \Omega_{2}$, we see that

$$
\left(\stackrel{\Delta}{\left.f_{1} \sigma_{1}\right)}\right)(\alpha)=\gamma f_{1}(w) \beta_{1} \in \Omega_{1} \text { and } \stackrel{\Delta}{\left(f_{2} \sigma_{2}\right)}(\alpha)=\gamma f_{2}(w) \beta_{1} \in \Omega_{2} .
$$

Hence, we arrive that $\alpha \in\left(\stackrel{\Delta}{f_{1} \sigma_{1}}\right)^{-1}\left(\Omega_{1}\right)$ and $\alpha \in\left(\stackrel{\Delta}{f_{2} \sigma_{2}}\right)^{-1}\left(\Omega_{2}\right)$, i.e., $\alpha \in\left(\stackrel{\Delta}{f_{1} \sigma_{1}}\right)^{-1}\left(\Omega_{1}\right) \cap\left(\stackrel{\Delta}{\left.f_{2} \sigma_{2}\right)^{-1}}\left(\Omega_{2}\right)\right.$.

Moreover, we show that $\stackrel{\Delta}{\tau_{1}} \sigma_{1}=\stackrel{\Delta}{\sigma_{2}} \tau_{2} \sigma_{2}$ on $\Pi$. Let $\alpha \in \Pi$. We obtain that $\sigma_{1}(\alpha) \in \Gamma W \Pi_{1}$. Substituting $\gamma w \beta_{1}$ for $\sigma_{1}(\alpha)$, where $\beta_{1} \in \Pi_{1}, w \in W, \gamma \in \Gamma$, we have

$$
\left(\tau_{1} \stackrel{\Delta}{f_{1} \sigma_{1}}\right)(\alpha)=\tau_{1}\left(\stackrel{\Delta}{f_{1}}\left(\sigma_{1}(\alpha)\right)\right)=\tau_{1}\left(\stackrel{\Delta}{f_{1}}\left(\gamma w \beta_{1}\right)\right)=\tau_{1}\left(\gamma f_{1}(w) \beta_{1}\right) .
$$

Using $\beta_{1} \in \Pi_{1} \subseteq \Omega_{1}$ and $\tau_{1}$ is a left $M \Gamma$-module homomorphism, we arrive that

$$
\tau_{1}\left(\gamma f_{1}(w) \beta_{1}\right)=\gamma f_{1}(w) \tau_{1}\left(\beta_{1}\right) .
$$

Since $\tau_{1}=\tau_{2}$ on $\Pi_{1}$ and $f_{1}=f_{2}$ on $W$, we find that

$$
\gamma f_{1}(w) \tau_{1}\left(\beta_{1}\right)=\gamma f_{2}(w) \tau_{2}\left(\beta_{1}\right)=\tau_{2}\left(\gamma f_{2}(w) \beta_{1}\right)=\tau_{2}\left(\stackrel{\Delta}{f_{2}}\left(\gamma w \beta_{1}\right)\right) .
$$

Using $\alpha \in \Pi_{2}$ and $\sigma_{1}=\sigma_{2}$ on $\Pi_{2}$, we see that

$$
\left(\tau_{2} \stackrel{\Delta}{f_{2}}\right)\left(\gamma w \beta_{1}\right)=\tau_{2}\left(\stackrel{\Delta}{f}\left(\sigma_{1}(\alpha)\right)\right)=\left(\tau_{2} \stackrel{\Delta}{f_{2} \sigma_{2}}\right)(\alpha) .
$$

Thus, we conclude that $\stackrel{\Delta}{\tau_{1}} \stackrel{\Delta}{f_{1} \sigma_{1}}=\stackrel{\Delta}{\tau_{2} \sigma_{2}}$ on $\Pi$, and so

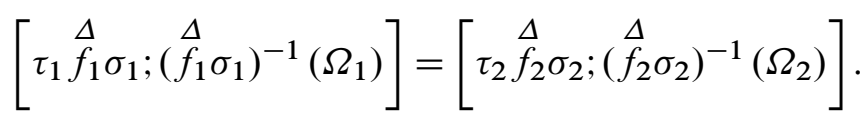


Consequently, multiplication is well defined. It is clearly that $[\tau ; \Omega][f ; U][\sigma ; \Lambda] \in \Delta$ for all $[f ; U] \in Q$ and $[\tau ; \Omega],[\sigma ; \Lambda] \in \Delta$.

Now we will show that $(Q, \Delta)$ is a gamma ring.

a) i) For all $[f ; U] \in Q$ and $[\tau ; \Omega],[\sigma ; \Lambda],[\delta ; \Sigma] \in \Delta$,

$$
\begin{aligned}
& ([\tau ; \Omega]+[\sigma ; \Lambda])[f ; U][\delta ; \Sigma]=[\tau+\sigma ; \Omega \cap \Lambda][f ; U][\delta ; \Sigma] \\
& =\left[(\tau+\sigma) \stackrel{\Delta}{f} \delta ;:(\stackrel{\Delta}{f} \delta)^{-1}(\Omega \cap \Lambda)\right] \\
& =\left[\begin{array}{c}
\Delta \\
\tau f \delta
\end{array} \stackrel{\Delta}{\sigma f} \delta ;(\stackrel{\Delta}{f} \delta)^{-1}(\Omega) \cap \stackrel{\Delta}{(f \delta)^{-1}(\Lambda)}\right] \\
& =\left[\begin{array}{c}
\Delta \\
\tau f
\end{array} ;(\stackrel{\Delta}{f} \delta)^{-1}(\Omega)\right]+\left[\begin{array}{c}
\Delta \\
\left.\sigma f \delta ;(\stackrel{\Delta}{f} \delta)^{-1}(\Lambda)\right]
\end{array}\right. \\
& =[\tau ; \Omega][f ; U][\delta ; \Sigma]+[\sigma ; \Lambda][f ; U][\delta ; \Sigma] \text {. }
\end{aligned}
$$

ii) For all $[f ; U],[g ; V] \in Q$ and $[\tau ; \Omega],[\sigma ; \Lambda] \in \Delta$,

$$
\begin{aligned}
{[\tau ; \Omega]([f ; U]+[g ; V])[\sigma ; \Lambda] } & =[\tau ; \Omega][f+g ; U \cap V][\sigma ; \Lambda] \\
& =\left[\tau(f+g) \sigma ;((f+g) \sigma)^{-1}(\Omega)\right],
\end{aligned}
$$

and

$$
\begin{aligned}
& {[\tau ; \Omega][f ; U][\sigma ; \Lambda]+[\tau ; \Omega][g ; V][\sigma ; \Lambda]} \\
& \left.=\left[\begin{array}{c}
\Delta \\
\tau f \sigma ;(f \sigma)^{-1}(\Omega)
\end{array}\right]+\left[\begin{array}{c}
\Delta \\
\tau \sigma \sigma
\end{array} \stackrel{\Delta}{g \sigma \sigma}\right)^{-1}(\Omega)\right]
\end{aligned}
$$

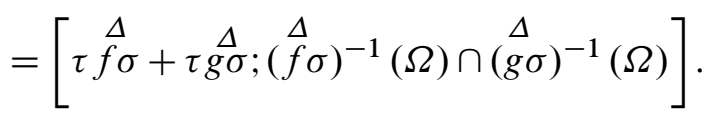

We will show that (2.6) and (2.8) are equivalent. Let $\left.\Pi=\stackrel{\Delta}{(f \sigma} \sigma)^{-1}(\Omega) \cap \stackrel{\Delta}{g} \sigma\right)^{-1}(\Omega)$ and $\alpha \in \Pi$. Then $\alpha \in(\stackrel{\Delta}{f} \sigma)^{-1}(\Omega)$ and $\left.\alpha \in \stackrel{\Delta}{g} \sigma\right)^{-1}(\Omega)$, i.e., $(\stackrel{\Delta}{f} \sigma)(\alpha) \in \Omega$ and $\stackrel{\Delta}{g} \sigma)(\alpha) \in \Omega$. Thus $(\stackrel{\Delta}{f} \sigma)(\alpha)+\stackrel{\Delta}{g} \sigma)(\alpha) \in \Omega$, and so $(\stackrel{\Delta}{f}+\stackrel{\Delta}{g}) \sigma(\alpha) \in \Omega$. That is $\alpha \in((f \stackrel{\Delta}{+} g) \sigma)^{-1}(\Omega)$. Then

$$
\left.\Pi \subseteq((f \stackrel{\Delta}{+} g) \sigma)^{-1}(\Omega) \cap(\stackrel{\Delta}{f} \sigma)^{-1}(\Omega) \cap \stackrel{\Delta}{g} \sigma\right)^{-1}(\Omega) .
$$

Moreover, for all $\alpha \in \Pi$

$$
\left(\tau\left(\begin{array}{c}
\Delta \\
+g
\end{array}\right) \sigma\right)(\alpha)=(\tau \stackrel{\Delta}{f} \sigma)(\alpha)+(\tau \stackrel{\Delta}{g \sigma})(\alpha)=(\tau \stackrel{\Delta}{f} \sigma+\tau \stackrel{\Delta}{g \sigma})(\alpha) .
$$

iii) For all $[f ; U] \in Q$ and $[\tau ; \Omega],[\sigma ; \Lambda],[\delta ; \Sigma] \in \Delta$,

$$
[\tau ; \Omega][f ; U]([\sigma ; \Lambda]+[\delta ; \Sigma])=[\tau ; \Omega][f ; U][\sigma+\delta ; \Lambda \cap \Sigma]
$$




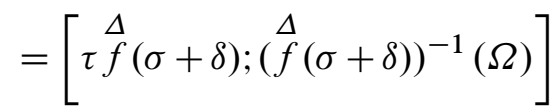

$$
\begin{aligned}
& \left.=\left[\begin{array}{c}
\Delta \\
\tau f \sigma
\end{array} \stackrel{\Delta}{\tau} f \delta ;(f \sigma \sigma)^{-1}(\Omega) \cap(\stackrel{\Delta}{f} \delta)\right)^{-1}(\Omega)\right] \\
& =\left[\begin{array}{c}
\Delta \\
\tau f \sigma ;(f \sigma)^{-1}(\Omega)
\end{array}\right]+\left[\begin{array}{c}
\Delta \\
\tau f \delta ;(f \delta))^{-1}(\Omega)
\end{array}\right] \\
& =[\tau ; \Omega][f ; U][\sigma ; \Lambda]+[\tau ; \Omega][f ; U][\delta ; \Sigma] \text {. }
\end{aligned}
$$

Set $\left.\Pi=(\stackrel{\Delta}{f} \sigma)^{-1}(\Omega) \cap(\stackrel{\Delta}{f} \delta)\right)^{-1}(\Omega)$, application of similar arguments yields the above equality.

b) i) For all $[f ; U],[g ; V] \in Q$ and $[\tau ; \Omega],[\sigma ; \Lambda],[\delta ; \Sigma] \in \Delta$,

$$
\begin{aligned}
& \left.([\tau ; \Omega][f ; U][\sigma ; \Lambda])[g ; V][\delta ; \Sigma]=[\stackrel{\Delta}{\tau f \sigma ;} \stackrel{\Delta}{f} \sigma)^{-1}(\Omega)\right][g ; V][\delta ; \Sigma] \\
& =\left[(\tau \stackrel{\Delta}{f} \sigma) \stackrel{\Delta}{g} \delta ;(\stackrel{\Delta}{g} \delta)^{-1}(\stackrel{\Delta}{f} \sigma)^{-1}(\Omega)\right] . \\
& \left.[\tau ; \Omega][f ; U]([\sigma ; \Lambda][g ; V][\delta ; \Sigma])=[\tau ; \Omega][f ; U][\sigma g \delta ;(g) \delta)^{-1}(\Lambda)\right] \\
& =\left[\stackrel{\Delta}{\tau} f(\sigma \stackrel{\Delta}{g} \delta) ;\left(\stackrel{\Delta}{f}\left(\sigma^{\Delta} g\right)\right)^{-1}(\Omega)\right]
\end{aligned}
$$

We will prove that (2.9) and (2.10) are equivalent. Let $\Pi=(\stackrel{\Delta}{g} \delta)^{-1}(\stackrel{\Delta}{f} \sigma)^{-1}(\Omega)$ and $\alpha \in \Pi$. Then $(\stackrel{\Delta}{g} \delta)(\alpha) \in(\stackrel{\Delta}{f} \sigma)^{-1}(\Omega)$, and so $(\stackrel{\Delta}{f} \sigma)(\stackrel{\Delta}{g} \delta)(\alpha) \in \Omega$. Hence, $\alpha \in$ $\left.\stackrel{\Delta}{(f}\left(\sigma \sigma_{g}^{\Delta} \delta\right)\right)^{-1}(\Omega)$. Thus $\left.\Pi \subseteq(\stackrel{\Delta}{g} \delta)^{-1}(\stackrel{\Delta}{f} \sigma)^{-1}(\Omega) \cap \stackrel{\Delta}{f}\left(\sigma^{\Delta} g \delta\right)\right)^{-1}(\Omega)$, and also

$$
((\tau \stackrel{\Delta}{f} \sigma) \stackrel{\Delta}{g} \delta)(\alpha)=(\tau \stackrel{\Delta}{f}(\sigma \stackrel{\Delta}{g} \delta))(\alpha), \text { for all } \alpha \in \Pi .
$$

ii) For all $[f ; U],[g ; V] \in Q$ and $[\tau ; \Omega],[\sigma ; \Lambda],[\delta ; \Sigma] \in \Delta$,

$$
\begin{aligned}
& ([\tau ; \Omega][f ; U][\sigma ; \Lambda])[g ; V][\delta ; \Sigma]=\left[\tau \stackrel{\Delta}{\tau} \sigma ;(\stackrel{\Delta}{f} \sigma)^{-1}(\Omega)\right][g ; V][\delta ; \Sigma] \\
& =\left[(\tau f \sigma \sigma) \stackrel{\Delta}{g} \delta ;(\stackrel{\Delta}{g} \delta)^{-1}(\stackrel{\Delta}{f} \sigma)^{-1}(\Omega)\right] \\
& =\left[\tau(\stackrel{\Delta}{f} \sigma \stackrel{\Delta}{g}) \delta ;(\stackrel{\Delta}{f} \sigma g \delta)^{-1}(\Omega)\right] \\
& =[\tau ; \Omega]\left[\stackrel{\Delta}{f} \sigma \stackrel{\Delta}{g} ;(\sigma \stackrel{\Delta}{g})^{-1}(U)\right][\delta ; \Sigma] \\
& =[\tau ; \Omega]([f ; U][\sigma ; \Lambda][g ; V])[\delta ; \Sigma] \text {. }
\end{aligned}
$$


Using similar arguments as above, we can prove.

c) Let $[\tau ; \Omega][f ; U][\sigma ; \Lambda]=[0 ; \Gamma]$ for all $[f ; U] \in Q$ and $[\tau ; \Omega],[\sigma ; \Lambda] \in \Delta$. For $\left[I_{\Gamma} ; \Gamma\right] \in \Delta$, where $I_{\Gamma}: \Gamma \rightarrow \Gamma$ is an identity left $M \Gamma$-module homomorphism, we see that

$$
\left[I_{\Gamma} ; \Gamma\right][f ; U]\left[I_{\Gamma} ; \Gamma\right]=[0 ; \Gamma],
$$

and so

$$
\left[\stackrel{\Delta}{I_{\Gamma}} \underset{f}{ } I_{\Gamma} ;\left(\stackrel{\Delta}{f} I_{\Gamma}\right)^{-1}(\Gamma)\right]=[0 ; \Gamma]
$$

Set $\Pi=\Gamma U \Gamma$. We first prove that $\left.\Gamma U \Gamma \subseteq \Gamma \cap \stackrel{\Delta}{f} I_{\Gamma}\right)^{-1}(\Gamma)$. For any $\gamma=\alpha u \beta \in$ $\Gamma U \Gamma$ where $u \in U, \alpha, \beta \in \Gamma$, we arrive at

$$
\left.\left.\stackrel{\Delta}{f} I_{\Gamma}\right)(\gamma)=\stackrel{\Delta}{f} I_{\Gamma}\right)(\alpha u \beta)=\stackrel{\Delta}{f}\left(I_{\Gamma}(\alpha u \beta)\right)=\stackrel{\Delta}{f}(\alpha u \beta)=\alpha f(u) \beta .
$$

We have $\left.\stackrel{\Delta}{f} I_{\Gamma}\right)(\gamma) \in \Gamma$, and so $\gamma \in\left(\stackrel{\Delta}{f} I_{\Gamma}\right)^{-1}(\Gamma)$. On the other hand, we get $\gamma \in \Gamma$. As a result $\left.\gamma \in \Gamma \cap \stackrel{\Delta}{f} I_{\Gamma}\right)^{-1}(\Gamma)$.

Now, for all $\gamma=\alpha u \beta \in \Gamma U \Gamma$ where $u \in U, \alpha, \beta \in \Gamma$,

$$
I_{\Gamma} \stackrel{\Delta}{f} I_{\Gamma}(\gamma)=I_{\Gamma} \stackrel{\Delta}{f} I_{\Gamma}(\alpha u \beta)=0(\alpha u \beta) .
$$

That is

$$
\alpha f(u) \beta=0, \text { for all } u \in U, \alpha, \beta \in \Gamma \text {. }
$$

Since $(M, \Gamma)$ is gamma ring, we get $f(u)=0$, for all $u \in U$, and so $f=0$. That is $[f ; U]=[0 ; M]$.

We arrive that $(Q, \Delta)$ is a gamma ring and we call it the maximal left gamma ring of quotient of $(M, \Gamma)$.

We proceed by showing that $(\Delta, Q)$ is characterized by certain reasonable properties that any gamma ring of quotient should have.

Remark 1 . Let $M$ be a $\Gamma$-ring, $\varepsilon \in \Gamma, e \in M$. If $(\varepsilon, e)$ is the strong right identity element of $(\Gamma, M)$, then $(e, \varepsilon)$ is the strong left identity element of $(M, \Gamma)$.

Proof. Assume that $(\varepsilon, e)$ is the strong right identity element of $(\Gamma, M)$. Thus we get $x \varepsilon e=x$ for all $x \in M$. For any $\gamma \in \Gamma, x, y \in M$, we have

$$
x(\varepsilon e \gamma-\gamma) y=x(\varepsilon e \gamma) y-x \gamma y=(x \varepsilon e) \gamma y-x \gamma y=x \gamma y-x \gamma y=0
$$

and so

$$
M(\varepsilon e \gamma-\gamma) M=(0) .
$$

Since $(\Gamma, M)$ is gamma ring, we get

$$
\varepsilon e \gamma=\gamma \text { for all } \gamma \in \Gamma \text {. }
$$


Using similar arguments as above, we can prove the followings:

Remark 2. Let $M$ be a $\Gamma$-ring, $\varepsilon \in \Gamma, e \in M$. If $(\varepsilon, e)$ is the strong left identity element of $(\Gamma, M)$, then $(e, \varepsilon)$ is the strong right identity element of $(M, \Gamma)$.

Let $(\varepsilon, e)$ is the strong right identity element of $(\Gamma, M)$. For a fixed element $a$ in $M$, consider a mapping $\lambda_{a \varepsilon}: M \rightarrow M$ defined by $\lambda_{a \varepsilon}(x)=a \varepsilon x$ for all $x \in M$. It is easy to prove that the mapping $\lambda_{a \varepsilon}$ is a right $\Gamma M$-module homomorphism. Moreover, for all $m \in M$,

$\lambda_{(a+b) \varepsilon}(m)=(a+b) \varepsilon m=a \varepsilon m+b \varepsilon m=\lambda_{a \varepsilon}(m)+\lambda_{b \varepsilon}(m)=\left(\lambda_{a \varepsilon}+\lambda_{b \varepsilon}\right)(m)$, and so

$$
\lambda_{(a+b) \varepsilon}=\left(\lambda_{a \varepsilon}+\lambda_{b \varepsilon}\right) .
$$

Now consider a mapping $\mu_{e \beta}: \Gamma \rightarrow \Gamma$ defined by $\mu_{e \beta}(\alpha)=\alpha e \beta$ for all $\alpha \in \Gamma$ is a left $M \Gamma$-module homomorphism. Indeed, we shown that $\Gamma$ is a left $M \Gamma$-module.

i) For all $\alpha, \gamma \in \Gamma$,

$$
\mu_{e \beta}(\alpha+\gamma)=(\alpha+\gamma) e \beta=\alpha e \beta+\gamma e \beta .
$$

ii) For all $\alpha, \gamma \in \Gamma$ and $m \in M$,

$$
\mu_{e \beta}(\alpha m \gamma)=(\alpha m \gamma) e \beta=\alpha m(\gamma e \beta)=\alpha m \mu_{e \beta}(\gamma) .
$$

Thus, $\mu_{e \beta}$ is a left $M \Gamma$-module homomorphism.

Using arguments as above, we can prove the followings:

$$
\mu_{e(\beta+\gamma)}=\mu_{e \beta}+\mu_{e \gamma} \text {. }
$$

Let

$$
\wp=\left\{\lambda_{a \varepsilon} \mid a \in M\right\} \text { and } \mho=\left\{\mu_{e \beta} \mid \beta \in \Gamma\right\} .
$$

These sets are additive groups and defining the mappings

$$
\wp \times \mho \times \wp \rightarrow \wp,\left(\lambda_{x \varepsilon}, \mu_{e \gamma}, \lambda_{y \varepsilon}\right) \mapsto \lambda_{x \varepsilon e \gamma y \varepsilon}=\lambda_{x \gamma y \varepsilon}
$$

and

$$
\mho \times \wp \times \mho \rightarrow \mho, \quad\left(\mu_{e \gamma}, \lambda_{x \varepsilon}, \mu_{e \beta}\right) \mapsto \mu_{e \gamma x \varepsilon e \beta}=\mu_{e \gamma x \beta} .
$$

It can be shown that $(\mho, \wp)$ is a gamma ring.

In the following theorem, $D(\Gamma, M)$ will denote collection of all dense ideal of $M$.

Theorem 3. Let $(\Gamma, M)$ be a semiprime gamma ring with strong right identity element. Then $(\Gamma, M)$ is a subring of $(\Delta, Q)$.

Proof. Consider the mappings

$$
\phi_{\varepsilon}: M \rightarrow Q, a \mapsto\left[\lambda_{a \varepsilon} ; M\right] \text { and } \psi_{e}: \Gamma \mapsto \Delta, \beta \rightarrow\left[\mu_{e \beta} ; \Gamma\right] .
$$

One readily checks that $\phi_{\varepsilon}$ is well defined. Using (2.11) equation, we have

$$
\begin{gathered}
\phi_{\varepsilon}(a+b)=\left[\lambda_{(a+b) \varepsilon} ; M\right]=\left[\lambda_{a \varepsilon} ; M\right]+\left[\lambda_{b \varepsilon} ; M\right]=\phi_{\varepsilon}(a)+\phi_{\varepsilon}(b), \\
\text { for all } a, b \in M .
\end{gathered}
$$


Hence, $\phi_{\varepsilon}$ is a group homomorphism. Now, we suppose that $\phi_{\varepsilon}(a)=\phi_{\varepsilon}(b)$. Then $\left[\lambda_{a \varepsilon} ; M\right]=\left[\lambda_{b \varepsilon} ; M\right]$, and so $\left(\lambda_{a \varepsilon} ; M\right) \sim\left(\lambda_{b \varepsilon} ; M\right)$. Hence there exists $W \subseteq M \cap M$ such that $W \in D(\Gamma, M)$ and $\lambda_{a \varepsilon}=\lambda_{b \varepsilon}$ on $W$, i.e., $a \varepsilon w=b \varepsilon w$, for all $w \in W$. That is $(a-b) \varepsilon W=(0)$. Since $W$ is a ideal of $M$, we have $(a-b) \varepsilon M \Gamma W=(0)$, and so $(a-b) \varepsilon M \in l_{\Gamma}(W)$. By Theorem $1(\mathrm{v})$, we get $(a-b) \varepsilon M=(0)$. In particular, taking $m$ by $e$, we get $(a-b) \varepsilon e=0$, and so $a=b$. Thus $\phi_{\varepsilon}$ is one-one. Hence $\phi_{\varepsilon}$ is a group monomorphism.

On the other hand, once easly checks that $\psi_{e}$ is a group monomorphism.

For all $\alpha, \beta \in \Gamma, m, n \in M$

$$
\phi_{\varepsilon}(m \beta n)=\left[\lambda_{(m \beta n) \varepsilon} ; M\right]=\left[\lambda_{m \varepsilon e \beta n \varepsilon} ; M\right],
$$

and

$$
\begin{gathered}
\phi_{\varepsilon}(m) \psi_{e}(\beta) \phi_{\varepsilon}(n)=\left[\lambda_{m \varepsilon} ; M\right]\left[\mu_{e \beta} ; \Gamma\right]\left[\lambda_{n \varepsilon} ; M\right] \\
=\left[\lambda_{m \varepsilon} \mu_{e \beta}^{\Delta} \lambda_{n \varepsilon} ;\left(\mu_{e \beta}^{\Delta} \lambda_{n \varepsilon}\right)^{-1}(M)\right] .
\end{gathered}
$$

We will show that (2.15) and (2) are equivalent. Set $W=M \Gamma M$ and $w=x \gamma y \in W$, where $\gamma \in \Gamma, x, y \in M$.

$$
\left.\stackrel{\Delta}{\left(\mu_{e \beta} \lambda_{n \varepsilon}\right.}\right)(w)=\left(\stackrel{\Delta}{\mu_{e \beta} \lambda_{n \varepsilon}}\right)(x \gamma y)=\stackrel{\Delta}{\mu_{e \beta}(n \varepsilon x \gamma y)}=n \varepsilon x \mu_{e \beta}(\gamma) y=n \varepsilon x \gamma e \beta y .
$$

Thus $\left(\stackrel{\Delta}{\mu_{e \beta} \lambda_{n \varepsilon}}\right)(w) \in M$, and so $w \in\left(\stackrel{\Delta}{\mu_{e \beta} \lambda_{n \varepsilon}}\right)^{-1}(M)$. Hence we obtain that $W \subseteq$ $\left(\mu_{e \beta} \lambda_{n \varepsilon}\right)^{-1}(M) \cap M$.

On the other hand, using (2.13), we conclude that

$$
\lambda_{m \varepsilon} \mu_{e \beta}^{\Delta} \lambda_{n \varepsilon}=\lambda_{m \varepsilon e \beta n \varepsilon}=\lambda_{(m \beta n) \varepsilon} .
$$

That is $\lambda_{m \varepsilon} \mu_{e \beta}^{\Delta} \lambda_{n \varepsilon}=\lambda_{(m \beta n) \varepsilon}$ on $W$. In that case, we get

$$
\phi_{\varepsilon}(m \beta n)=\phi_{\varepsilon}(m) \psi_{e}(\beta) \phi_{\varepsilon}(n) .
$$

Using similar arguments as above, we can shown that

$$
\psi_{e}(\alpha m \gamma)=\psi_{e}(\alpha) \phi_{\varepsilon}(m) \psi_{e}(\gamma) .
$$

Hence $\left(\psi_{e}, \phi_{\varepsilon}\right)$ is a gamma ring monomorphism, and so $(\Gamma, M)$ is a subring of $(\Delta, Q)$.

Theorem 4. Let $(\Gamma, M)$ be a semiprime gamma ring with strong left identity element. $(\Delta, Q)$ satisfies the following properties:

i) If $U \in D(\Gamma, M)$ and $f: U \rightarrow M$ is a right $\Gamma M$-module homomorphism, then there exists an element $q \in Q$ such that $f(u)=q \varepsilon u$ for all $u \in U$.

ii) For all $q \in Q$ there exists $U \in D(\Gamma, M)$ such that $q \varepsilon U \subseteq M$.

iii) For all $q \in Q$ and $U \in D(\Gamma, M), q \varepsilon U=(0)$ if and only if $q=0$. 
Proof. i) Let $q \in Q, u \in U, f: U \rightarrow M$ be a right $\Gamma M$-module homomorphism and $q=[f ; U]$. Since $M$ can be embedded in $Q$, we can write $u=\left[\lambda_{u \varepsilon} ; M\right]$ such that $\lambda_{u \varepsilon}: M \rightarrow M, x \mapsto u \varepsilon x$ for all $u \in U$. Then we have

$$
q \varepsilon u=[f ; U]\left[\mu_{e \varepsilon} ; \Gamma\right]\left[\lambda_{u \varepsilon} ; M\right]=\left[f \stackrel{\Delta}{\mu_{e \varepsilon}} \lambda_{u \varepsilon} ;\left(\mu_{e \varepsilon}^{\Delta} \lambda_{u \varepsilon}\right)^{-1}(U)\right]
$$

and

$$
f(u)=\left[\lambda_{f(u) \varepsilon} ; M\right] .
$$

We will show that (2.17) and (2.18) are equivalent. Indeed, for $W=U \Gamma M$ and $x=y \gamma m \in W$, where $y \in U, \gamma \in \Gamma, m \in M$, we have

$$
\begin{gathered}
\stackrel{\Delta}{\left(\mu_{e \varepsilon} \lambda_{u \varepsilon}\right)(x)=\left(\mu_{e \varepsilon} \lambda_{u \varepsilon}\right)(y \gamma m)=\mu_{e \varepsilon}}(u \varepsilon y \gamma m) \\
=u \varepsilon y \mu_{e \varepsilon}(\gamma) m=u \varepsilon y \gamma e \varepsilon m=u \varepsilon y \gamma m \in U .
\end{gathered}
$$

In this way, we get $x=y \gamma m \in\left(\stackrel{\Delta}{\mu_{e \varepsilon}} \lambda_{u \varepsilon}\right)^{-1}(U)$, and so $W \subseteq\left(\mu_{e \varepsilon} \lambda_{u \varepsilon}\right)^{-1}(U) \cap M$. Moreover, for all $x \in W$, we get

$$
\begin{aligned}
& \left(f \stackrel{\Delta}{\mu_{e \varepsilon} \lambda_{u \varepsilon}}\right)(x)=\left(f \stackrel{\Delta}{\mu_{e \varepsilon} \lambda_{u \varepsilon}}\right)(y \gamma m)=\left(f \stackrel{\Delta}{\mu_{e \varepsilon}}\right)(u \varepsilon y \gamma m)
\end{aligned}
$$

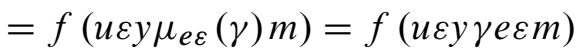

$$
\begin{aligned}
& =f(u \varepsilon y \gamma m)=f(u) \varepsilon y \gamma m \\
& =\lambda_{f(u) \varepsilon}(y \gamma m)=\lambda_{f(u) \varepsilon}(x) \text {. }
\end{aligned}
$$

That is $f \stackrel{\Delta}{\mu} \mu_{e \varepsilon} \lambda_{u \varepsilon}=\lambda_{f(u) \varepsilon}$ on $W$.

Therefore, there exists an element $q \in Q$ such that $f(u)=q \varepsilon u$ for all $u \in U$.

ii) Let $q \in Q$. Then there exists $U \in D(\Gamma, M)$ such that $q=[f ; U]$ and $f: U \rightarrow M$ be a right $\Gamma M$-module homomorphism. According to $(i)$, we have $f(u)=q \varepsilon u$ for all $u \in U$. Thus $q \varepsilon U \subseteq M$. Consequently, there exists $U \in D(\Gamma, M)$ such that $q \varepsilon U \subseteq M$.

iii) Let $q \in Q$ and $U \in D(\Gamma, M), q \varepsilon U=(0)$. Then $q=[f$; $U]$. If $q \varepsilon U=(0)$, then $q \varepsilon u=0$, for all $u \in U$. By $(i), f(u)=q \varepsilon u=0$, for all $u \in U$. Thus $f(u)=0$, for all $u \in U$, and so $q=[0 ; U]=[0 ; M]=0 Q$. Conversely, let $q=0$. Then $q=$ $[f ; U]=[0 ; M]$, and so $(f ; U) \sim(0 ; M)$. That is $f(u)=0$ for all $u \in U \subseteq M$. According to $(i), f(u)=q \varepsilon u=0$ for all $u \in U$, so $q \varepsilon U=(0)$. This completes the proof.

\section{REFERENCES}

[1] S. A. Amitsur, “On rings of quotients,” Sympos. math., vol. 8, pp. 149-164, 1972.

[2] W. E. Barnes, “On the $\Gamma$-rings of Nobusawa," Pac. J. Math., vol. 18, pp. 411-422, 1966.

[3] K. I. Beidar, W. S. Martindale III, and A. V. Mikhalev, Rings with generalized identities, ser. Pure and Applied Mathematics. New York: Marcel Dekker, 1996, vol. 196.

[4] A. Genç, "The quotient rings of prime $\Gamma$-rings (Asal $\Gamma$-halkalarının kesirler halkası," Ph.D. dissertation, Ege University, Institute of Science, Bornova Izmir, 2008. 
[5] S. Kyuno, Gamma rings, ser. Hadronic Press Monographs in Mathematics. Palm Harbor: Hadronic Press, Inc., 1991.

[6] W. S. Martindale III, "Prime rings satisfying a generalized polynomial identity," J. Algebra, vol. 12, pp. 576-584, 1969.

[7] N. Nobusawa, "On a generalization of the ring theory," Osaka J. Math., vol. 1, pp. 81-89, 1964.

[8] M. Öztürk and Y. B. Jun, "On the centroid of the prime gamma rings. ii," Turk. J. Math., vol. 25, no. 3, pp. 367-377, 2001.

[9] M. A. Öztürk and Y. B. Jun, "On the centroid of the prime gamma rings," Commun. Korean Math. Soc., vol. 15, no. 3, pp. 469-479, 2000.

[10] M. A. Öztürk and Y. B. Jun, "Regularity of the generalized centroid of semi-prime Gamma rings," Commun. Korean Math. Soc., vol. 19, no. 2, pp. 233-242, 2004.

[11] M. A. Öztürk and H. Yazarli, "Modules over the generalized centroid of semi-prime gamma rings," Bull. Korean Math. Soc., vol. 44, no. 2, pp. 203-213, 2007.

[12] Y. Utumi, "On quotient rings," Osaka Math. J., vol. 8, pp. 1-18, 1956.

\section{Authors' addresses}

\section{Emine Koc}

Cumhuriyet University, Faculty of Science, Department of Mathematics, 58140 Sivas, TURKEY

E-mail address: eminekoc@cumhuriyet.edu.tr

\section{Öznur Gölbaşı}

Cumhuriyet University, Faculty of Science, Department of Mathematics, 58140 Sivas, TURKEY

E-mail address: ogolbasiecumhuriyet.edu.tr 